\title{
Detection of Cavernous transformation of the portal vein in a 22-year-old male with upper gastrointestinal bleeding and anemia by Abdominal Duplex Ultrasonography. A Case Report.
}

\author{
Joseph Arkorful ${ }^{1}$, Theophilus Adu - Bredu ${ }^{2}$, and Obed Otoo ${ }^{1}$ \\ ${ }^{1}$ Kwame Nkrumah University of Science and Technology \\ ${ }^{2}$ Komfo Anokye Teaching Hospital
}

July 18, 2021

\begin{abstract}
Cavernous transformation of the portal vein (CTPV) is a rare condition characterized by the formation of multiple tortuous tiny blood vessels within and around a portal vein which has been occluded by thrombus. Early diagnosis of CTPV is relevant for timely intervention which could save the liver from non-reversible damage
\end{abstract}

Title: Detection of Cavernous transformation of the portal vein in a 22-year-old male with upper gastrointestinal bleeding and anemia by Abdominal Duplex Ultrasonography. A Case Report.

Running Head : Sonographic detection of Cavernous transformation of the portal vein

Authors : Joseph Arkorful ${ }^{1,2}$, Theophilus Kofi Adu - Bredu ${ }^{2,3}$, Obed Otoo ${ }^{2}$.

1. University Hospital, Kwame Nkrumah University of Science and Technology, Kumasi, Ghana

2. Department of Medical Diagnostics, College of Health Sciences, Kwame Nkrumah University of Science and Technology, Kumasi, Ghana

3. Department of Obstetrics and Gynecology, Komfo Anokye Teaching Hospital, Kumasi, Ghana

Corresponding Author: Joseph Arkorful. Postal Address: P.O. Box UP 1717, K.N.U.S.T - Kumasi. Tel: +233540604576 Email: josepharkorful@hotmail.com

\section{ABSTRACT}

Cavernous transformation of the portal vein (CTPV) is a rare condition characterized by the formation of multiple tortuous tiny blood vessels within and around a portal vein which has been occluded by thrombus. Early diagnosis of CTPV is relevant for timely intervention which could save the liver from end stage disease. However, the diagnosis is often difficult due to its rarity and diverse clinical symptoms. Ultrasonography could serve as a reliable modality in the early diagnosis of this condition to improve patients' outcomes. This case report narrates the role of Abdominal Duplex ultrasonography in the diagnosis of CTPV in a 22-year-old male presenting with upper gastrointestinal (GI) bleeding and anemia.

\section{KEY CLINICAL MESSAGE}

CTPV; a rare condition can be missed clinically due to its non-specific symptoms. CTPV should hence be part of the differential diagnosis for patients who present with upper GI bleeding and anemia; and the utilization of Abdominal Duplex ultrasound encouraged since it helps in detection of CTPV to ensure adequate patientcare. 


\section{KEYWORDS:}

Cavernous transformation of the Portal vein, Duplex Ultrasonography, Portal hypertension, Upper gastrointestinal bleeding, Portal cavernoma.

\section{INTRODUCTION}

Cavernous transformation of the portal vein (CTPV) also known as portal cavernoma is a very rare condition of the liver which is usually characterized by the formation of multiple tortuous tiny blood vessels within and around a portal vein which has been partially or completely occluded by a thrombus ${ }^{1-3}$.

Liver cirrhosis, hepatoma, splenectomy, liver transplant and pancreatitis are known causes of portal vein thrombosis that subsequently leads to CTPV in adults ${ }^{1}$. However, CTPV have been mostly recorded in individuals with long-term thrombosis of the portal vein without evidence of primary liver disease ${ }^{1-3}$. Cases of CTPV have also been recorded in individuals with hematological diseases ${ }^{4}$ and those with primary systemic vascular abnormalities which have resulted in chronic portal vein thrombosis ${ }^{1,2,5}$.

The initial diagnosis of CTPV by clinicians may be challenging due to its diverse causes and clinical presentations ${ }^{4,6}$. Common clinical manifestations are however those associated with portal hypertension as a result of chronic portal vein thrombosis; such as upper gastrointestinal bleeding (secondary to esophageal varices), anemia, splenomegaly, and ascites ${ }^{3}$.

Abdominal Duplex Ultrasonography is a useful first-line imaging tool for both adult and pediatric patients with clinical presentations pointing towards the suspicion of liver disease or portal hypertension; because it allows for examination of abdominal organs and their vascular architecture in a real-time, efficient and safe manner ${ }^{7}$. Computed Tomography Angiography (CTA) and Magnetic Resonance Angiography (MRA) may be used for the confirmation of CTPV when an initial diagnosis is made with ultrasonography.

We therefore report on a case of a 22-year-old male in whom a diagnosis of cavernous transformation of the portal vein was made with Duplex ultrasonography who presented clinically with anemia and hematemesis.

\section{CASE REPORT}

A 22-year-old male presented to the emergency room of our hospital with slight dizziness, four episodes of vomiting mixed with blood and passage of dark stool over a 48-hour period. The patient had no prior history of chronic alcoholism, liver cirrhosis or hepatoma. According to the patient, he has had an episode of vomiting of blood and abdominal pain a year ago and was treated and discharged on account of bleeding peptic ulcer disease. This current episode of vomiting of blood with slight dizziness occurred again and was brought to our hospital by his parents for further management.

Upon physical examination, patient appeared pale with no evidence of respiratory distress. There was no complaints of cough, chills, headache or fever. No obvious abdominal mass palpated.

Vital signs: Blood pressure: $143 / 96 \mathrm{mmHg}$, Heart rate: 100beats/minute, Temperature: $36.9^{\circ} \mathrm{C}$, Respiratory rate: 15 breaths/minute, Oxygen saturation: $97 \%$.

Based on the patient's clinical presentation, an initial diagnosis of bleeding peptic ulcer disease with differential diagnosis of Mallory-Weiss tear was made.

Laboratory investigation was then initiated and highlighted as follows:

There was evidence of severe anemia (low hemoglobin and red blood cells (RBC)), low platelets, low hematocrit (HCT) and low mean corpuscular volume (MCV) upon full blood count investigation

Findings:

Hemoglobin: 5g/dl (Reference range: $13.0-17.0 \mathrm{~g} / \mathrm{dl}$ ); RBC: 3.09 (Reference range: 4.2 - 6.0

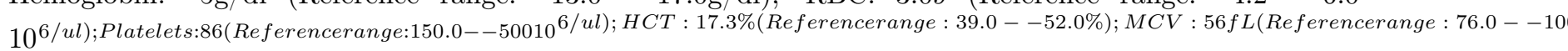


FIGURE 1. Sonogram demonstrating an occluded portal vein (arrow head) with noticeable surrounding formed tortuous blood vessels (arrows) at the porta hepatis

Also noted was diffuse echogenic gallbladder wall which demonstrated tiny hepatopetal vascular channels under color Doppler interrogation.

Liver was however of average size with no surface nodularity or discrete masses (Figure 2).

Also noted was an enlarged spleen with multiple dilated tortuous blood vessels at the region of the splenic hilum which were suggestive of portal hypertension (Figure 2 and Figure 3)

Mild ascites was also detected.

Pancreas and both kidneys were within normal limits.

No intra-abdominal masses detected.

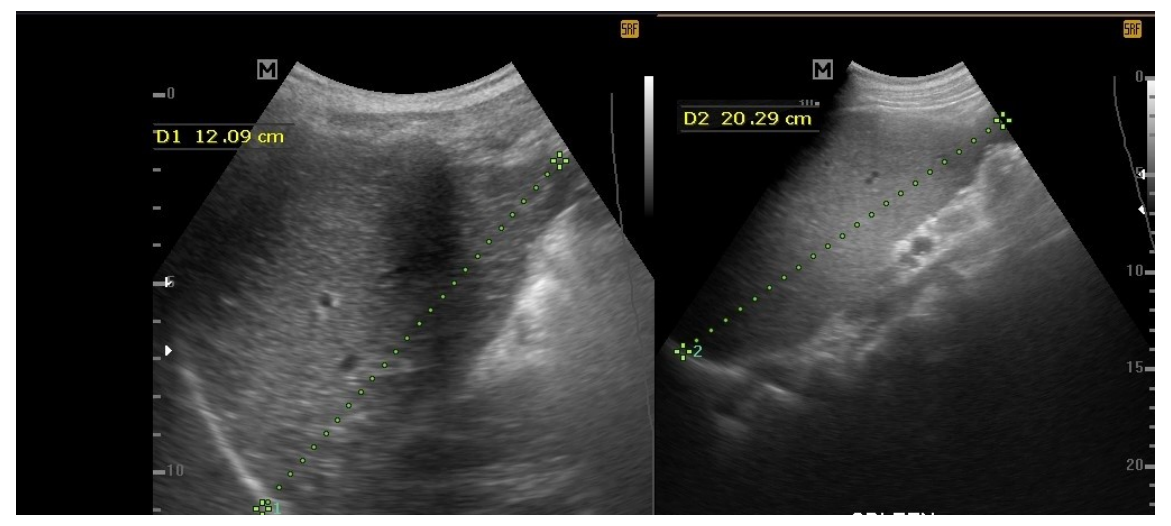

FIGURE 2. Sonograms demonstrating a homogenous-appearing liver (A) and an Enlarged spleen measuring about $20.3 \mathrm{~cm}$ in length (B)

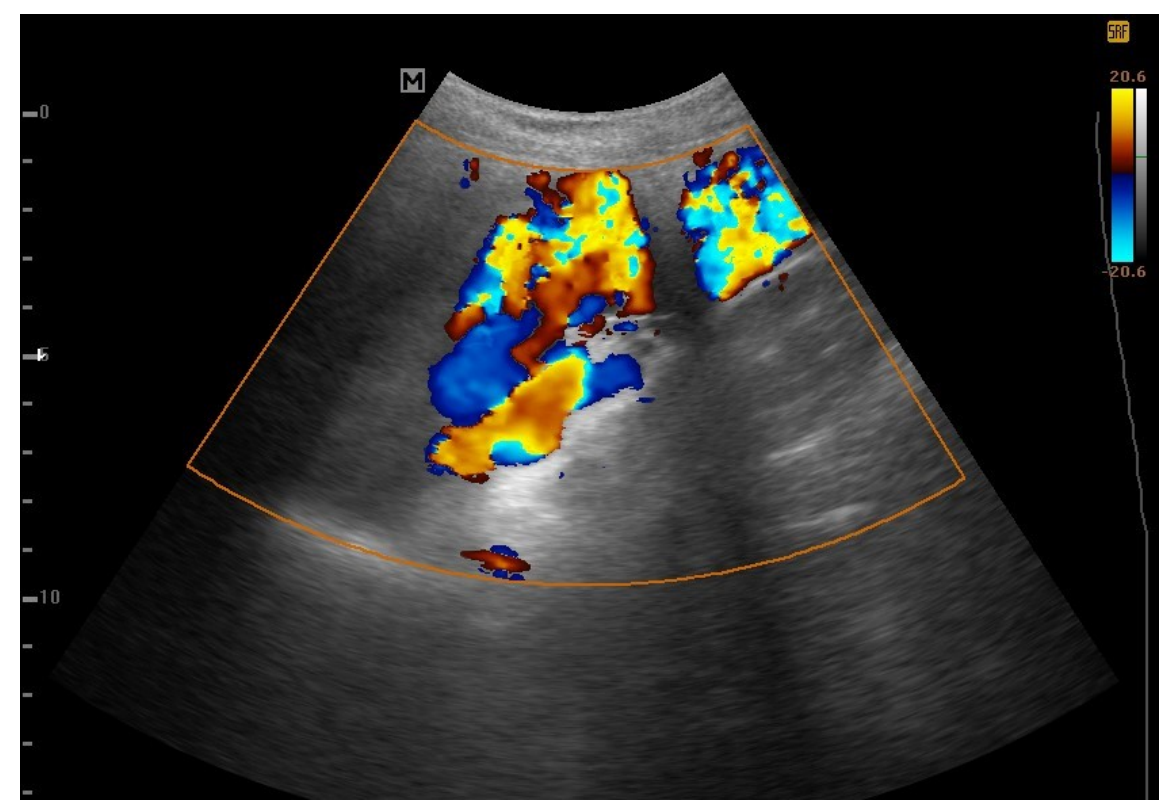


FIGURE 3. Sonogram showing a Spleen $(\mathrm{S})$ with multiple dilated tortuous blood vessels at the region of the splenic hilum (arrow heads).

An MRI or CT scan was not performed for this patient because the Abdominal Ultrasound findings, the patient's clinical history and examinations were enough to make the diagnosis of CTPV.

Follow up 83 Further investigation

Patient was hemotransfused with 1 unit of blood on day of admission and subsequently 2 unit of blood and oral iron supplements to manage his anemia. A repeat Hemoglobin count 3 days after depicted an increase of hemoglobin count from $5 \mathrm{~g} / \mathrm{dL}$ to $7.9 \mathrm{~g} / \mathrm{dL}$.

An upper gastrointestinal (GI) endoscopy was then requested for further evaluation after the patient was stabilized.

The upper GI endoscopy revealed esophageal varices with mild extension of the varices to the gastric fundus. Normal appearance of the duodenum identified.

Patient was managed on oral blood supplements to further correct his anemia and was discharged from the hospital 2 weeks after. During his time of admission, he did not experience any of the initial symptoms he presented to the hospital with. Also, there was improvement in his hemoglobin count $(\mathrm{Hb}=11.2 \mathrm{~g} / \mathrm{dL})$; with no complaints of dizziness, vomiting of blood or passage of dark-colored stool at the time of discharge.

He was then scheduled for 2 weekly review for further management by both the medical and surgical teams on out-patient basis.

\section{DISCUSSION}

Cavernous transformation of the portal vein (CTPV) is a very rare condition usually characterized by tiny tortuous blood vessels formed around a thrombosed portal vein ${ }^{3}$. This condition is mostly accompanied by portal hypertension and the formation of porto-systemic collaterals and varices ${ }^{8}$. CTPV have been mostly recorded in individuals with long-term thrombosis of the portal vein without evidence of liver cirrhosis or hepatoma ${ }^{1-3}$.

In CTPV, two types of collateral circulations are observed namely portoportal and portosystemic. In the event of the occurrence of portal vein thrombosis, re - canalization usually takes place in patients without cirrhosis or any liver disease which causes an increased resistance to blood flow ${ }^{6}$. This leads to increase in hepatic arterial blood flow at the acute phase and mesenteric collaterals compensates for the obstruction. Subsequently, there is dilatation of collateral vascular channels within the hepatoduodenal and hepatocolic ligaments, around common bile duct, hepatic ducts and gall bladder draining in to the intrahepatic portal veins to bypass the obstruction ${ }^{9}$; and this was evident in our case where gallbladder wall thickening with evidence of pericholecystic varices under Color Doppler interrogation were observed. This "venous rescue" process continues and subsequently organizes into a cavernous transformation in about $3-5$ weeks. This compensatory process however has deleterious effect on intestinal circulation as it is known to be associated with bowel ischemia ${ }^{6}$. When the portal cavernoma persists for a long time, the collaterals encase and compress the biliary tree which causes damage to the intra and/or extra hepatobiliary ducts and this may lead to loss of functional liver tissue.

Clinically, most patients affected with CTPV present with symptoms such as hematemesis and melena secondary to possible bleeding esophageal varices ${ }^{6,8}$. These symptoms may however be non-specific for CTPV since patients with gastrointestinal disease conditions may also present with hematemesis and melena; and this was observed in our case where the patient's initial diagnosis was directed towards bleeding peptic ulcer and likely Mallory-Weiss tear. Other signs associated with portal hypertension such as anemia, splenomegaly, ascites and abnormally low platelets count (thrombocytopenia) have also been reported in patients with $\mathrm{CTPV}^{8}$. All these aforementioned clinical symptoms of CTPV were evident in the patient in our case study. 
Administering of medication and surgery are known interventions for the management of CTPV; with the success of treatment depending on the etiology of the disease ${ }^{4}$. The surgical procedure which involves the creation of shunts decreases the portal pressure, enables hepatopetal blood from portal vein to the liver and also alleviate the abnormal hemodynamic status in the portal system ${ }^{10}$. This have been known to be associated with favorable outcomes.

In our case study, the etiology of the CTPV could not be determined since the patient had no history of liver cirrhosis, hepatoma or pancreatitis. A chronic portal vein thrombosis from a likely systemic vascular disorder could be the cause of the CTPV.

\section{CONCLUSION}

Ultrasonography remains the first line radiological modality for assessing the abdominal organs in most centers in the world due to its accessibility and low cost. Available literature suggest ultrasonography as a reliable modality in diagnosing this pathology; with CT angiogram and MRI serving as complimentary modalities for confirmation of CTPV.

Medical practitioners must therefore include CTPV as part of their differential diagnosis in patients who present with upper GI bleeding symptoms and anemia especially those without prior history or clinical suspicions of liver cirrhosis, hepatoma or pancreatitis. Duplex Abdominal ultrasonography must hence be encouraged in the initial diagnostic workup in such patients.

In view of this, sonographers and radiologists must pay close attention to the hepatic vasculature especially the segmental portal venous system when scanning patients presenting with upper GI bleeding and anemia. This could allow for early detection of CTPV if present since early diagnosis is crucial in improving patients' outcomes. Early sonographic detection of this pathology is also necessary for appropriate timely intervention which could save the liver from end stage disease.

ACKNOWLEDGEMENTS: The authors extend gratitude to all those who helped in the preparation of this work.

PATIENT CONSENT FOR PUBLICATION: Obtained

CONFLICT OF INTEREST: The authors declare no conflict of interest

AUTHORS CONTRIBUTION: JA performed the ultrasound examination, conceived the idea and involved in the initial preparation of the manuscript. TKA-B and OO contributed to writing of the manuscript.

\section{REFERENCES}

1. De Gaetano AM, Lafortune M, Patriquin H, et al. Cavernous transformation of the portal vein: patterns of intrahepatic and splanchnic collateral circulation detected with Doppler sonography. AJR Am J Roentgenol . 1995;165(5):1151-1155.

2. Kumar A, Sharma P, Arora A. Review article: portal vein obstruction - epidemiology, pathogenesis, natural history, prognosis and treatment.Alimentary Pharmacology 83 Therapeutics . 2015;41(3):276-292.

3. Salihefendić N, Licanin Z, Zildzić M. [Cavernous transformation of portal vein]. Med Arh . 2005;59(2):132134.

4. Chang C-Y, Yang P-M, Hung S-P, et al. Cavernous transformation of the portal vein: etiology determines the outcome.Hepatogastroenterology . 2006;53(72):892-897.

5. Harmanci O, Bayraktar Y. Portal hypertension due to portal venous thrombosis: etiology, clinical outcomes. World J Gastroenterol . 2007;13(18):2535-2540.

6. Ramos R, Park Y, Shazad G, A.Garcia C, Cohen R. Cavernous Transformation of Portal Vein Secondary to Portal Vein Thrombosis: A Case Report. J Clin Med Res . 2012;4(1):81-84. 
7. Coley BD. Pediatric applications of abdominal vascular Doppler imaging: Part I. Pediatr Radiol . 2004;34(10):757-771.

8. Sorrentino D, Labombarda A, Debiase F, Trevisi A, Giagu P. Cavernous transformation of the portal vein associated to multiorgan developmental abnormalities. Liver Int . 2004;24(1):80-83.

9. Nasim M, Majid B, Tahir F, Majid Z, Irfan I. Cavernous Transformation of Portal Vein in the Setting of Protein C and Anti-thrombin III Deficiency. Cureus . 2019;11(9).

10. Zhang H, Zhang N, Li M, Jin W, Pan S. Surgical Treatment of Portal Vein Cavernous Transformation. World J Surg . 2004;28(7):708-711. 